\title{
Suppression of PGE2 production via disruption of MAPK phosphorylation by unsymmetrical dicarbonyl curcumin derivatives
}

\begin{abstract}
Curcumin is an important molecule found in turmeric plants and has been reported to exhibit some profound anti-inflammatory activities by interacting with several important molecular targets found in the mitogen-activated protein kinase and NF- $\kappa \beta$ pathways. As part of our continuing effort to search for new anti-inflammatory agents with better in vitro and in vivo efficacies, we have synthesized a series of new unsymmetrical dicarbonyl curcumin derivatives and tested their effects on prostaglandin E2 secretion level in interferon$\gamma /$ lipopolysaccharide-activated macrophage cells. Among those, five compounds exhibited remarkable suppression on prostaglandin E2 production with IC50 values ranging from 0.87 to $18.41 \mu \mathrm{M}$. The most potent compound $17 \mathrm{f}$ was found to down-regulate the expression of cyclooxygenase- 2 mRNA suggesting that this series of compounds could possibly target the mitogen-activated protein kinase signal transduction pathway. Whilst the compound did not affect the expression of the conventional mitogen-activated protein kinases, the results suggest that it could disrupt the phosphorylation and activation of the proteins particularly the c-Jun N-terminal kinases. Finally, the binding interactions were examined using the molecular docking and dynamics simulation approaches.
\end{abstract}

Keyword: Prostaglandin E2; COX-2 mRNA expression; MAPK phosphorylation; Molecular; Dynamic simulation; Unsymmetrical dicarbonyl curcumin derivatives 